\title{
Evidence for a lineage of virulent bacteriophages that target Campylobacter
}

\author{
Andrew R Timms ${ }^{1 *}$, Joanna Cambray-Young ${ }^{2}$, Andrew E Scott ${ }^{1,4}$, Nicola K Petty ${ }^{3}$, Phillippa L Connerton ${ }^{1}$, \\ Louise Clarke ${ }^{3}$, Kathy Seeger ${ }^{3}$, Mike Quail ${ }^{3}$, Nicola Cummings ${ }^{1}$, Duncan J Maskell ${ }^{2}$, Nicholas R Thomson ${ }^{3}$, \\ lan F Connerton ${ }^{1}$
}

\begin{abstract}
Background: Our understanding of the dynamics of genome stability versus gene flux within bacteriophage lineages is limited. Recently, there has been a renewed interest in the use of bacteriophages as 'therapeutic' agents; a prerequisite for their use in such therapies is a thorough understanding of their genetic complement, genome stability and their ecology to avoid the dissemination or mobilisation of phage or bacterial virulence and toxin genes. Campylobacter, a food-borne pathogen, is one of the organisms for which the use of bacteriophage is being considered to reduce human exposure to this organism.

Results: Sequencing and genome analysis was performed for two Campylobacter bacteriophages. The genomes were extremely similar at the nucleotide level $(\geq 96 \%)$ with most differences accounted for by novel insertion sequences, DNA methylases and an approximately $10 \mathrm{~kb}$ contiguous region of metabolic genes that were dissimilar at the sequence level but similar in gene function between the two phages. Both bacteriophages contained a large number of radical S-adenosylmethionine (SAM) genes, presumably involved in boosting host metabolism during infection, as well as evidence that many genes had been acquired from a wide range of bacterial species. Further bacteriophages, from the UK Campylobacter typing set, were screened for the presence of bacteriophage structural genes, DNA methylases, mobile genetic elements and regulatory genes identified from the genome sequences. The results indicate that many of these bacteriophages are related, with 10 out of 15 showing some relationship to the sequenced genomes.

Conclusions: Two large virulent Campylobacter bacteriophages were found to show very high levels of sequence conservation despite separation in time and place of isolation. The bacteriophages show adaptations to their host and possess genes that may enhance Campylobacter metabolism, potentially advantaging both the bacteriophage and its host. Genetic conservation has been shown to extend to other Campylobacter bacteriophages, forming a highly conserved lineage of bacteriophages that predate upon campylobacters and indicating that highly adapted bacteriophage genomes can be stable over prolonged periods of time.
\end{abstract}

\section{Background}

Bacteriophages (phages) are naturally occurring predators of bacteria that are ubiquitous in the environment; they are almost certainly the most abundant biological entities on the planet [1]. It is commonly accepted that phage genomes are an extremely rich source of novel and unique DNA sequences, and that phage genomes are highly variable, often showing a mosaic patchwork

\footnotetext{
* Correspondence: andrew.timms@nottingham.ac.uk

'Division of Food Sciences, School of Biosciences, University of Nottingham, Sutton Bonington Campus, Loughborough, Leicestershire, LE12 5RD, UK
}

of genetic segments acquired from a range of sources including other phages and bacteria. However, this view is almost certainly an oversimplification; as more phage genomes become available it is clear that closely related phage lineages exist in the environment which may be stable over appreciable time-scales and geographic areas $[2,3]$. Understanding how these lineages evolve and adapt to their hosts will provide useful insights into the phage pan-genome, genetic flux within communities and phage genome stability.

The general availability of phages in the environment coupled with their ease of isolation and cultivation has

\section{Biomed Central}


led them to be used in a variety of ways. Phages have been instrumental in the development of molecular biology, their use as typing tools for bacterial pathogens continues today and recently there has been a resurgence of interest in phage intervention to control pathogens, so called 'bacteriophage therapy'. The closely related zoonotic pathogens Campylobacter jejuni and Campylobacter coli are major causes of infectious bacterial gastroenteritis in humans [4-6], and have been associated with rare but serious, sometimes fatal, neurological sequelae such as Guillain-Barré syndrome, Miller-Fisher syndrome and the onset of reactive arthritis [7-9]. Using phages to reduce Campylobacter at multiple stages of the food chain is a promising sustainable intervention strategy but requires detailed knowledge of phage genomes at the sequence level. Although previous studies have shown that the application of phages can effectively reduce Campylobacter contamination [10-12], to date the most common use of Campylobacter phages has been in typing schemes allowing the discrimination between different Campylobacter isolates [13,14]. Previous studies have shown that all the Campylobacter phages examined to date belong to either the Myoviridae or Siphoviridae tailed phage families [15]. Based on genome size, they have been categorized into three groups; group III 130 - $140 \mathrm{~kb}$, group II 180 - $190 \mathrm{~kb}$ and the typing phages NCTC 12676 and NCTC 12677, which are reported to be $\sim 320 \mathrm{~kb}$ in size, as group I $[16,17]$. Molecular characterization of these phages has been slow, with many of the currently available Campylobacter phages being extremely refractory to genomic analysis. In many cases phage genomic DNA is resistant to digestion with any of the standard restriction endonucleases, although, HhaI has proven to be useful to discriminate some group III phages [16-18]. However, thorough characterization of Campylobacter specific phages (indeed any phage intended for therapeutic applications) is a prerequisite to avoid the inadvertent transfer or mobilization of harmful genes [19].

In this work we report the first full genome sequences, analysis of virion proteins and the genome analysis for two large virulent Campylobacter specific phages; CP220 a phage isolated from chickens in $2003[10,20]$ and
CPt10 a member of the UK Campylobacter typing scheme phages, isolated from a slaughterhouse environment prior to 1989 [21]. Both display typical genome sizes for group II phages, a morphology typical of the Myoviridae phages $[17,20]$ and exhibit broad but different host ranges with both phages notably able to lyse Campylobacter jejuni and Campylobacter coli isolates. The genome sequences illuminate a very highly conserved Campylobacter specific phage lineage that has survived the ongoing competition between host and virus.

\section{Results and Discussion}

\section{Genome structure}

Two virulent Campylobacter phages have been sequenced to reveal double stranded DNA genomes of 177493 bp (CP220) and 175720 bp (CPt10), a comparison of the genomes can be found in Table 1. The full annotated genome sequences have been deposited with the EMBL nucleotide sequence database with accession numbers; CP220 [EMBL: FN667788] and CPt10 [EMBL: FN667789]. These phages have a GC content comparable to that found in the host species, 30.5\%, 30.3\% and $31.1 \%$ for C. jejuni NCTC 11168, RM1221 and C. coli RM2228 respectively [22]. Both the CP220 and CPt10 genomes show a distinct strand bias for putative protein coding sequences (CDS), (see Additional file 1 - Table S1) with the majority of the CDSs lying on the 'forward' strand, an observation in accordance with that for many other phages where a similar bias in orientation is observed [23,24]. In many bacterial species predominant gene orientation is frequently parallel to the direction of replication of the forward strand, thus minimising interference between RNA transcription and DNA replication. However, both phage genomes contain notable exceptions to this strand bias, including two distinct sections of DNA where contiguous runs of CDSs lie on the reverse strand (Figure 1).

In most cases the strand alignment of the open reading frames is supported by the apparent change in strand AG content, with a distinct preference for A and G bases in the coding strand. This bias was also reflected in the codon usage [25]. Comparison of the codon usage frequencies from the entire genome sequences of

Table 1 Comparison of basic parameters for CP220 and CPt10 phage genomes

\begin{tabular}{|c|c|c|c|c|c|c|}
\hline & Genome size bpa (+/- variable repeats) & $\begin{array}{c}\text { Genome } \\
\% \text { G+C } \\
\text { content }\end{array}$ & $\begin{array}{c}\text { CDS } \\
\% \text { G+C } \\
\text { content }\end{array}$ & $\begin{array}{c}\text { Number of } \\
\text { CDS }^{\text {b }}\end{array}$ & $\begin{array}{c}\text { Coding } \\
\text { Density }^{\mathrm{b}} \%\end{array}$ & $\begin{array}{c}\text { Strand Bias } \\
\text { 'forward' }\end{array}$ \\
\hline CP220 & 177 493/171841 & 27.4 & 28.2 & 194 & 88.4 & $173 / 194$ \\
\hline CPt10 & 175 720/173 299 & 27.3 & 28.0 & 201 & 89.7 & $180 / 201$ \\
\hline
\end{tabular}




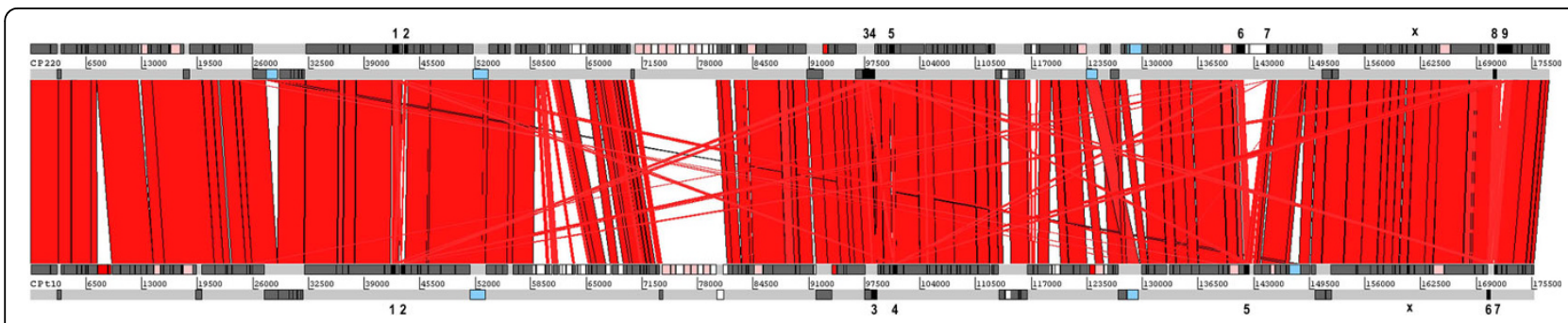

Figure 1 Genome alignments of CP220 and CPt10. Nucleotide alignment of the virulent bacteriophages CP220 (top) and CPt10 (bottom) generated using the Artemis Comparison Tool (ACT). The CDS regions are indicated by bars top and bottom showing the 'forward' and 'reverse' strands. CDS regions are coloured according to the following key: grey - areas of nucleotide identity, white - significantly different CDS regions present in only one of the genomes, solid black - repeat regions (numbered according to the current genome annotation), red - DNA modification proteins, blue - insertion elements, pink - radical SAM proteins. Red bars indicate regions of sequence homology, the diagonal lines link multiple repeat regions in both genomes indicating the conserved nature of these sequences throughout both genomes.

CP220 and CPt10 and from several C. jejuni and C. coli genome sequences reveals that in cases where synonymous codons are available, the phages show a preference for alternative codons to those employed by campylobacters (Additional file 2). There is a small but definite bias towards codons with $\mathrm{A}$ or $\mathrm{U}$ at the third position, which probably reflects the high $\mathrm{A}+\mathrm{T}$ content of these phage genomes, although as noted above the phage genomes closely match that of host campylobacters in terms of their overall base composition.

The phages carry tandem tRNA genes with arginine and tyrosine type anticodons. Unusually, the Tyr-tRNA in CP220 shows a single base substitution in a highly conserved tRNA residue but it is not clear how this base change would impact on function. Examination of the phage codon usage shows that the two codons recognized by these tRNAs are represented more frequently in the phage coding regions than in Campylobacter, and thus probably explains the evolutionary pressure for their retention. What is perhaps more surprising is that these phages don't carry more tRNA genes, for example T4 encodes eight tRNAs [26], as there are a number of other codons that are represented more frequently in phage CDSs than in Campylobacter. The codon preferences observed could be an evolutionary relic, considering the wide range of organisms that appear to have contributed to the phage genomes, or it may be a strategy to fully utilize the available tRNA pools in Campylobacter to maintain key host functions whilst translating phage proteins necessary for replication and reproduction.

Of the 201 and 194 predicted CDSs identified for $\mathrm{CPt} 10$ and CP220 respectively, some show significant conservation (greater than $20 \%$ identity at the protein level) to a range of structural and enzymatic proteins from phage T4 (Table 2). Proteins Gp20 (portal vertex protein) and $\mathrm{Gp} 23$ (the major capsid protein) are both represented in the Campylobacter phage sequences with identity to the corresponding T4 proteins of $28 \%$ and
$31 \%$ respectively. The observation of sequence conservation suggests that these phages belong to the diverse T4-like phage superfamily but somewhat distant to the archetypal member of the group. The overall architecture of the genome appears to follow a modular construction, with most of the phage structural and DNA replication genes clustered in the left and right arms of the genomes as represented in Figure 1, while the central third of the genome comprises a much more heterogeneous selection of genes involved with metabolic processes. However, in common with other phage genome sequences, a large proportion of putative CDSs 85/ 194 from CP220 and 95/201 from CPt10 show no significant matches to sequences currently deposited in databases.

Direct comparison of the two genomes reveals an extremely high level of conservation (Figure 1), with an average nucleotide identity over the entire length of the phage chromosomes of $96.2 \%$. So high is the conservation between these two phages it is possible to identify the boundaries of insertion or deletion events encompassing discrete CDSs. Comparison of the genomes revealed that 26 CDSs are unique to CP220 and 28 are unique to $\mathrm{CPt} 10$. The majority of these have no significant matches to database sequences, however, two of the unique sequences in CPt10 code for putative DNA methyltranferases not present in CP220. CPt10_0091 shares $59 \%$ identity (over 588 amino acids) with a type III restriction/modification methyltranferase subunit from Campylobacter jejuni. Although the gene has a confirmed frame-shift mutation in a poly-adenosine tract towards the distal end of the gene, the full-length product could still be expressed through a process of transcriptional slippage $[27,28]$. The second putative methyltranferase, CPt10_1471, shows similarity to DNA methyltranferases from both Clostridium and Campylobacter sp. CP220 and CPt10 have different Campylobacter host ranges (data not shown), the two identified methyltranferases may enable CPt10 to avoid host 
Table 2 Comparison of T4 and CP220 Structural Proteins

\begin{tabular}{|c|c|c|c|c|}
\hline $\begin{array}{l}\text { CP220 } \\
\text { CDS }\end{array}$ & $\begin{array}{c}\text { T4 } \\
\text { Length }^{\mathrm{a}}\end{array}$ & $\begin{array}{l}\text { CP220 } \\
\text { Length }^{\mathrm{a}}\end{array}$ & T4 Gene Function ${ }^{b}$ & $\begin{array}{l}\text { CP220/T4 Identity } \\
(\%) \\
\text { E value }^{c} \\
\end{array}$ \\
\hline CPT_0001 & 610 & 744 & $\begin{array}{l}\text { Gp17 Terminase subunit with nuclease and ATPase activity; binds single-stranded DNA, } \\
\text { Gp16 and Gp20 }\end{array}$ & $\begin{array}{l}143 / 436(32 \%) \\
\mathrm{E}=4 \mathrm{e}^{-47}\end{array}$ \\
\hline CPT_0005 & 516 & 503 & Gp39 DNA topoisomerase large subunit & $\begin{array}{l}186 / 516(35 \%) \\
E=4 e^{-73}\end{array}$ \\
\hline CPT_0009 & 342 & 347 & Gp61 DNA primase subunit & $\begin{array}{l}73 / 330(22 \%) \\
0.017\end{array}$ \\
\hline CPT_0010 & 319 & 304 & Gp44 Clamp loader subunit, DNA polymerase accessory protein & $\begin{array}{l}95 / 319(29 \%) \\
\mathrm{E}=8 \mathrm{e}^{-27}\end{array}$ \\
\hline$\overline{\text { CPT_0011 }}$ & 305 & 352 & RNaseH ribonuclease & $\begin{array}{l}56 / 151(37 \%) \\
E=6 e^{-16}\end{array}$ \\
\hline CPT_0029 & 157 & 178 & Gp49 EndoVII packaging and recombination endonuclease VII & $\begin{array}{l}28 / 108(25 \%) \\
E=0.11\end{array}$ \\
\hline CPT_0030 & 524 & 569 & Gp20 Portal vertex protein of head & $\begin{array}{l}130 / 456(28 \%) \\
E=5 e^{-40}\end{array}$ \\
\hline CPT_0033 & 487 & 429 & Gp30 DNA ligase & $\begin{array}{l}128 / 484(26 \%) \\
E=2 e^{-23}\end{array}$ \\
\hline CPT_0034 & 659 & 516 & Gp18 Tail sheath monomer & $\begin{array}{l}58 / 235(24 \%) \\
E=3 e^{-04}\end{array}$ \\
\hline CPT_0037 & 134 & 120 & Gp25 Baseplate wedge subunit & $\begin{array}{l}26 / 66(39 \%) \\
\mathrm{E}=2 \mathrm{e}^{-07}\end{array}$ \\
\hline$\overline{C P T \_0041}$ & 660 & 1214 & Gp6 Baseplate wedge subunit & $\begin{array}{l}86 / 389(22 \%) \\
E=0.025\end{array}$ \\
\hline CPT_0045 & 163 & 252 & Gp19 Tail tube protein & $\begin{array}{l}49 / 176(27 \%) \\
E=9 e^{-11}\end{array}$ \\
\hline CPT_0046 & 150 & 158 & Gp4 Head completion protein & $\begin{array}{l}44 / 147(29 \%) \\
E=5 e^{-08}\end{array}$ \\
\hline CPT_0048 & 185 & 255 & Gp55 Sigma factor for T4 late transcription & $\begin{array}{l}33 / 123(26 \%) \\
E=0.37\end{array}$ \\
\hline CPT_0051 & 521 & 444 & Gp23 Major head protein & $\begin{array}{l}131 / 412(31 \%) \\
E=2 e^{-41}\end{array}$ \\
\hline CPT_0053 & 659 & 578 & Gp18 Tail sheath protein & $\begin{array}{l}149 / 436(34 \%) \\
E=1 e^{-51}\end{array}$ \\
\hline CPT_0058 & 163 & 196 & Gp19 Tail tube protein & $\begin{array}{l}41 / 170(24 \%) \\
E=5 e^{-09}\end{array}$ \\
\hline CPT_0115 & 898 & 882 & Gp43 DNA polymerase & $\begin{array}{l}244 / 901(27 \%) \\
E=4 e^{-56}\end{array}$ \\
\hline CPT_0125 & 475 & 445 & Gp41 DNA primase-helicase subunit & $\begin{array}{l}120 / 453(26 \%) \\
E=1 e^{-34}\end{array}$ \\
\hline CPT_0148 & 587 & 472 & UvsW RNA-DNA and DNA-DNA helicase, ATPase & $\begin{array}{l}124 / 407(30 \%) \\
E=5 e^{-42}\end{array}$ \\
\hline CPT_0174 & 272 & 456 & Gp15 Tail sheath stabilizer and completion protein & $\begin{array}{l}54 / 214(25 \%) \\
E=1 e^{-05}\end{array}$ \\
\hline CPT_0177 & 301 & 306 & Gp32 Single-stranded DNA binding protein & $\begin{array}{l}65 / 212(30 \%) \\
E=6 e^{-12}\end{array}$ \\
\hline CPT_0181 & 374 & 361 & RnIA RNA ligase 1 and tail fiber attachment catalyst & $\begin{array}{l}80 / 297(26 \%) \\
E=3 e^{-10}\end{array}$ \\
\hline CPT_0193 & 212 & 236 & Gp21 Prohead core protein protease & $\begin{array}{l}52 / 153(33 \%) \\
E=2 e^{-08}\end{array}$ \\
\hline
\end{tabular}

a Length refers to the number of amino-acid residues in the CDS and are calculated from the genomic sequence

b $\mathrm{T} 4$ gene product and functions are according to Miller [26]

${ }^{c}$ Identity and E values are from NCBI BLAST results, performed on $5^{\text {th }}$ January 2009 
restriction defences in some strains through modification of its own DNA. In addition methylation is known to be involved in control of gene expression in many organisms and these enzymes may be involved in such a process during CPt10 infection, either through modification of its own DNA or by modification and possibly silencing of host gene expression.

The largest region differentiating these two phages is a cluster encoding 10 CDSs in CPt10, and 12 in CP220. The two clusters show little relationship at the nucleotide or amino-acid sequence level but show remarkable functional conservation, where five CDSs from each cluster possess conserved radical $S$-adenosylmethionine (SAM) domains and each cluster appears to posses a putative glycine amidinotransferase. These regions are unlikely to have diverged from a common progenitor as this would require localised mutation rates in these regions to be much higher than in the surrounding phage genome. It is more likely these sequences have been acquired en masse from a related organism, whether phage or bacterial, by homologous or nonhomologous recombination.

In total there are 11 proteins belonging to the radical SAM superfamily in CP220 and 12 in CPt10. Radical SAM proteins are involved in the cleavage of unreactive $\mathrm{C}-\mathrm{H}$ bonds in a range of biochemical processes as diverse as DNA repair, lysine metabolism and the generation of vitamins and cofactors such as biotin, heme and thiamine [29]. The presence of so many radical SAM proteins in these phage genomes is highly unusual. Approximately 27,000 proteins have so far been assigned to this superfamily, of these the overwhelming majority $(\sim 22,800)$ are bacterial proteins, with only around 22 having been identified in phage genomes thus far [30]. These proteins are associated with oxygen-independent oxidation reactions and are often found in anaerobic organisms; presumably they may also confer a metabolic advantage at low oxygen tensions, precisely the conditions that Campylobacter faces in the gut lumen. This analysis identifies a central portion of both phages, carrying five radical SAM genes, as being an interchangeable module with related functions that are likely to be non-essential for phage proliferation. However, it is possible that these metabolic enzymes could temporarily improve the competitive fitness of infected Campylobacter thus also benefiting the phage.

\section{Repeat regions and genome expansion}

CP220 and CPt10 both carry regions of repetitive DNA, accounting for $3.2 \%$ (at 10 loci) and $1.4 \%$ (at 8 loci) of their respective genomes. In all but one instance, common to both phages, the repeat regions are extragenic. The sole exception being a $21 \mathrm{bp}$ repeat motif found within the putative coding sequences CPT_0180 and
CPt10_1891 in CP220 and CPt10 respectively. The remaining repetitive regions contain between 3 and 17 copies of an approximately 75 bp core repeat unit (Additional file 1 - Table S2), with minor sequence polymorphisms evident between units both within and between repeat regions. PCR was used to further examine these repeats in CP220. Six out of the seven regions examined were shown to produce amplicons of mixed lengths, despite the template originating from a single plaque purified population of CP220 (Figure 2). Thus, the length of these regions would appear to be subject to variation during phage proliferation and may serve to create genome diversity. Examination of individual repeat regions shows the presence of tandem repeats containing the same sequence polymorphisms, suggesting that expansion (or contraction) could occur by slipstrand mispairing during DNA replication. Other repeat regions show evidence that recombination or translocation of repeat units has occurred, for example sequences more characteristic of repeat units within regions 6 and 8 are present in repeat region 9 of CP220, so either identical sequence changes have occurred at multiple sites or more likely the repeat units have propagated themselves in the phage genome.

The generation of genomes of variable length could be an adaptive mechanism of the phages in response to conditions in their host or environment, perhaps by influencing the packaged genome and gene dosage in the terminally redundant regions. However, it is difficult to see to what extent these relatively small changes in genome size would contribute to such a mechanism. More likely, they may serve as recombination substrates

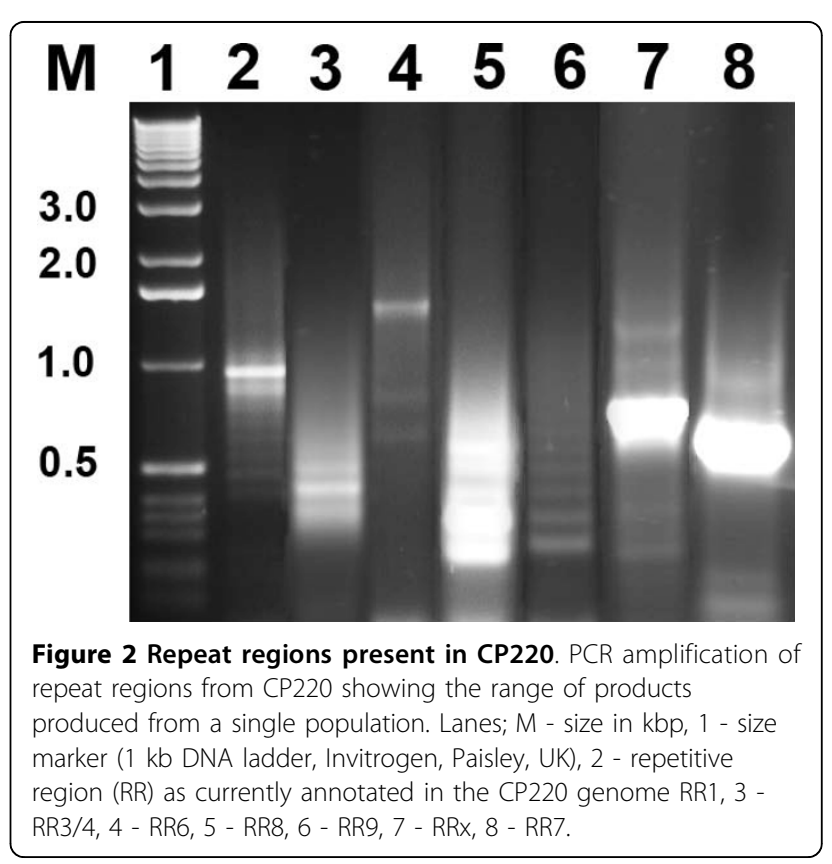


allowing reassortment of phage genes. For example, there are three instances in CP220 (flanking CPT_0046, CPT_0110 to CPT_0113 and CPT_0159 to CPT_0160), where one or more discrete CDSs are bordered by repeat sequences. The relative positions of the repeat regions within the CP220 and CPt10 genomes are generally conserved (Figure 1). Thus, they could also facilitate the exchange of larger segments between closely related phages. It is clear however that any CP220 and by corollary CPt10 recovered, is actually but a single representative of a larger family of related genomes under continual size-flux, the relative proportions of which may be dictated by selective environmental pressures.

\section{Tail proteins}

Both CP220 and CPt10 have multiple genes whose products show homology to Gp18 - the tail sheath and Gp19 - tail tube proteins. Protein sequencing of tryptic fragments of proteins excised from SDS-PAGE demonstrates that at least two types of Gp18 and two types of Gp19 are present in mature CP220 virions (Figure 3). It is unknown whether the individual protein types are mixed within a single phage virion or if discrete populations exist, with each mature virion containing a single protein type. Regardless, these variants arise within virus populations originally propagated from single plaques. The versions of Gp18 are associated with mobile elements; this suggests a mechanism whereby copies of the tail sheath genes may be exchanged, thereby enabling reassortment between related phages and possibly modulating their host range.

\section{Phage replication and packaging}

The phages carry genes involved in replication including; putative genes for DNA primase, sliding clamp, sliding clamp loader proteins, DNA polymerase, $\mathrm{RNaseH}$ and DNA ligase, thus replication of the phages is most likely independent of the host replication complex. Several putative transcription factors are present and may coordinate gene expression throughout the phages lifecycle; these genes provide inviting targets to examine transcriptional control during the infection process in these phages.

Putative terminases with significant similarity to the T4 large terminase subunit Gp17, 32\% identity (143/436 amino acids) for CP220, have been identified in both phages. Interestingly, the CP220 protein exhibits sequence conservation within the C-terminal domain, responsible for the DNA nuclease activity, but not the $\mathrm{N}$-terminus which has an ATPase function and is responsible for DNA binding [31]. CP220 also encodes a putative single-strand DNA binding protein and T4-like endonuclease VII packaging and recombination enzyme,

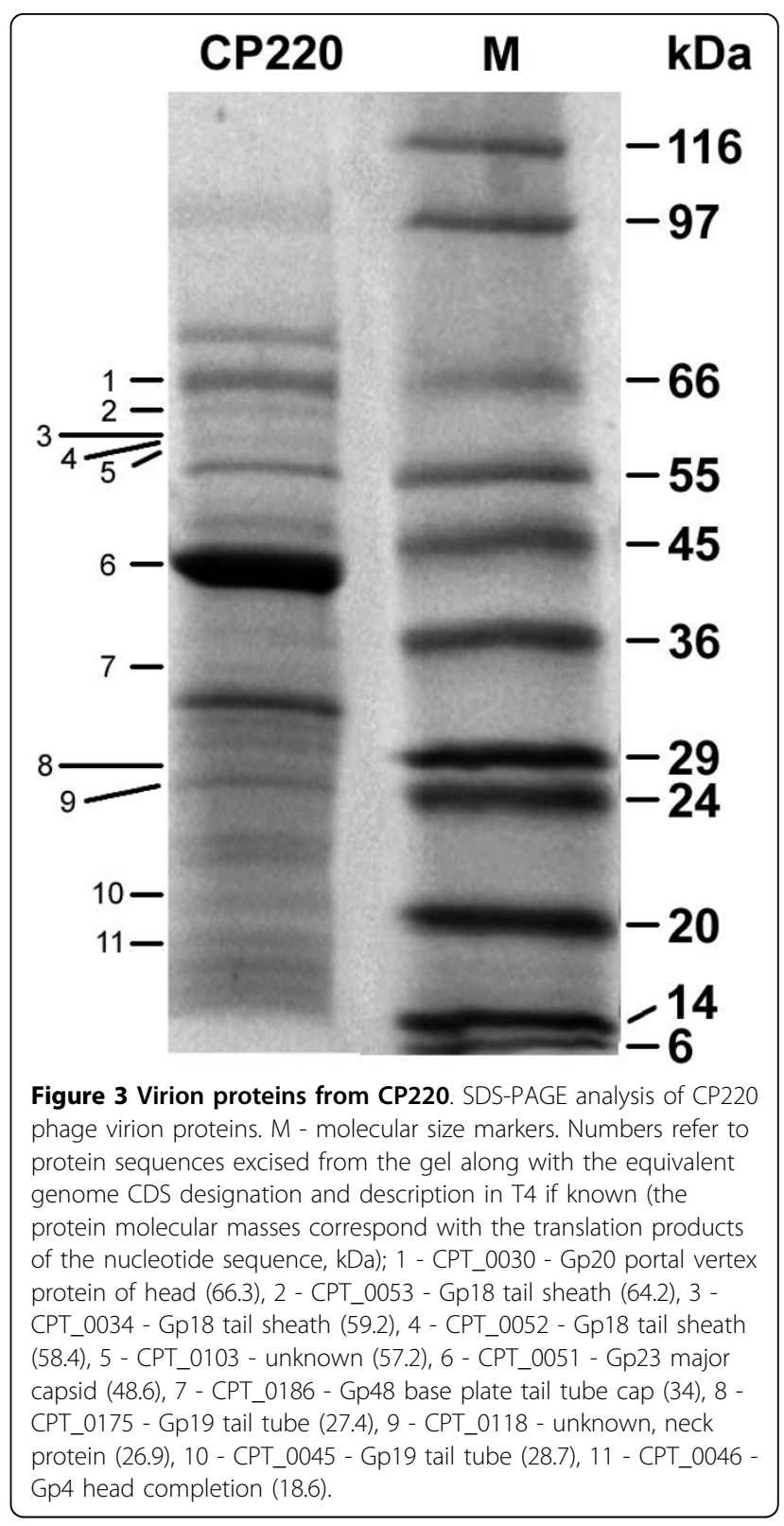

each of which have been shown to interact with Gp17 during T4 genome packaging.

\section{Inter and intra-genomic gene flux}

Four new insertion sequence (IS) elements, ISCaje1-4, were identified in the genomes of these two phages, all belonging to the IS200/IS605 family. In total five separate IS elements were found in the two genomes, four of which were closely related to each other and belong to the subgroup that encode only TnpB. ISCaje1 was found in both CP220 and CPt10, but the two isoforms, which share $97 \%$ DNA identity, have inserted into different locations in each genome. ISCaje2 in CPt10 and ISCaje3 in CP220 show similarity to each other, but are 
sufficiently different to constitute discrete IS elements (less than 95\% identity at the DNA level). Although they were found to insert at the same target sequence (ATTTT), their relative genomic locations were different. The presence of related IS elements in both phage genomes strongly suggests that these phages are derived from the same lineage. They predate upon the same range of hosts, thus allowing the transfer of related insertion elements to occur between phages or between host and phage genomes. The fourth novel IS element, ISCaje4, represents a unique insertion in the CP220 genome, and encodes a putative TnpA subunit in addition to TnpB (subgroup IS608).

Whether these insertion elements are active during infection is unknown, but they may provide mechanisms whereby regions of the phage genomes can be mobilised, perhaps allowing integration of DNA sequences into the host chromosome or conversely the integration of novel genes into the phage genomes. The presence of putative homing endonucleases at similar sites in both genomes may also provide a partial exclusion mechanism during infection of multiple phage types, or provide a means by which transfer of those genes surrounding the endonuclease can occur, predominantly in favour of the homing endonuclease carrying phage $[32,33]$.

Genetic transfer between host and virulent phage genomes can occur; illegitimate recombination allows the integration of host DNA and if the integrated fragments carry genes that are useful or advantageous to the phage, or to hosts that the phage subsequently infect, they may be maintained in the genome. There is certainly evidence that genes from diverse sources have been integrated into both CP220 and CPt10. Fifteen CDSs in CP220 show significant homology to Campylobacter and its taxonomic relatives Helicobacter, Arcobacter and Wolinella (Table 3). The presence of such DNA provides an indication of the importance of phage in the transfer and dissemination of genes between related species as, presumably, if these genes can mobilise onto the phage genome the reverse could also be true. Although during the course of these experiments the gene contents have remained fixed.

The products of other CDSs encoded by these phages showed the highest level of sequence identity with those from Clostridium sp., Bacillus sp., Porphyromonas sp. and Fusobacterium sp. Thus, the host range of this phage may not have been limited to C. jejuni and C. coli but extended to other genera. Consistent with this notion, these organisms do share common niches with various Campylobacter sp. and therefore provide opportunities for encounters between phage and bacteria to occur.
Rohwer has estimated that there are as many as 2 billion phage associated open reading frames still to be discovered and potentially 100 million different phage genotypes [34]. However, with the availability of an expanded portfolio of phage genome sequences it has become evident that while there are undoubtedly a large number of unique phage genomes, there are many other cases where phages can be clustered into closely related phylogenetic groups $[3,35]$. That these two phages have maintained their genomes in an almost identical configuration and at such a high level of sequence conservation, despite their independent isolation 14 years apart, suggests that they are well adapted to their hosts; we presume that this is Campylobacter, and that just as host bacterial genomes are under selection by their environment there are also significant selective pressures to maintain optimal phage genome structure.

\section{Distribution of CP220 and CPt10 CDSs in other virulent phage targeting Campylobacter}

To explore the possibility that these two bacteriophages were part of a conserved lineage targeting Campylobacter, we compared a Campylobacter phage (CP8) that we had partially sequenced years previously. CP8 had been isolated from chickens independently of both CP220 and $\mathrm{CPt} 10$ but unlike the latter had proved refractory to further molecular analysis [10]. Nevertheless CP8 exhibits significant sequence identity with CP220, ranging from $56 \%$ up to $100 \%$ over a total aggregate sequence length of $11470 \mathrm{bp}$. We have also extended the current analysis by designing primers for specific regions within CP220 and CPt10 that feature insertion sequences, methylases and structural genes. These primer sets have been used in PCR experiments with genomic DNA isolated from the remaining 15 phages of the UK Campylobacter typing scheme.

Five of the phages did not yield DNA amplicons with any of the primer combinations tested $(\varphi 1, \varphi 4, \varphi 7, \varphi 12$ and $\varphi 16)$, either due to gene absence, sequence divergence or DNA modifications that prevent efficient PCR amplification [36,37]. However, the remaining 10 phages, of disparate origin, share sequence loci with CP220 and CPt10 (Additional file 3). The typing phages have previously been classified on the basis of genome size by Sails et al [17]. Based on this classification CP220 and CPt10 fall within group II, and it is clear from Additional file 3 that the majority of the phages that contain amplicon homologues also belong to group II. Phages 8, 14 and 15 display particularly good correlation with the genes present in CP220 and CPt10. However, $\varphi 6$ isolated in the USA and belonging to group III, also shares at least four genes with the CP220/CPt10 family and in this respect is similar to CP8, which is 
Table 3 Putative CP220 CDSs showing homology to proteins in Campylobacter and closely related species Helicobacter, Arcobacter and Wolinella

\begin{tabular}{|c|c|c|}
\hline CP220 CDS & $\begin{array}{l}\text { CP220 } \\
\text { Length }^{\mathrm{a}}\end{array}$ & $\begin{array}{l}\text { Source Organism, Function }{ }^{b} \\
\text { Length }^{a} \text {, Identity }(\%)(\text { E value })^{c}\end{array}$ \\
\hline CPT_0017 & 264 & $\begin{array}{l}\text { Arcobacter butzleri RM4018.; Radical SAM domain protein } \\
254,115 / 252(45 \%)\left(2 \mathrm{e}^{-53}\right)\end{array}$ \\
\hline CPT_0040 & 70 & $\begin{array}{l}\text { Helicobacter hepaticus ATCC 51449; Hypothetical protein HH0764 } \\
62,22 / 57(38 \%)\left(4 \mathrm{e}^{-04}\right)\end{array}$ \\
\hline CPT_0066 & 328 & $\begin{array}{l}\text { Campylobacter upsaliensis RM3195.; Hypothetical protein CUPA0063 } \\
305,83 / 296(28 \%)\left(2 \mathrm{e}^{-17}\right)\end{array}$ \\
\hline CPT_0084 & 342 & $\begin{array}{l}\text { Campylobacter fetus subsp. fetus 82-40; Heme biosynthesis protein, putative } \\
354,45 / 178(25 \%)\left(1 e^{-07}\right)\end{array}$ \\
\hline CPT_0095 & 144 & $\begin{array}{l}\text { Campylobacter jejuni RM1221; Putative lipoprotein } \\
125,45 / 120(37 \%)\left(2 e^{-12}\right)\end{array}$ \\
\hline CPT_0105 & 202 & $\begin{array}{l}\text { Campylobacter curvus 525.92; hypothetical protein CCV52592_0059 } \\
189,109 / 200(54 \%)\left(1 e^{-51}\right)\end{array}$ \\
\hline CPT_0123 & 143 & $\begin{array}{l}\text { Helicobacter hepaticus ATCC 51449.; Starvation-inducible DNA-binding protein Dps } \\
156,42 / 140(30 \%)\left(4 \mathrm{e}^{-12}\right)\end{array}$ \\
\hline CPT_0127 & 205 & $\begin{array}{l}\text { Campylobacter lari RM2100; Hypothetical protein CLA1217 } \\
211,66 / 109(60 \%)\left(2 \mathrm{e}^{-32}\right)\end{array}$ \\
\hline CPT_0137 & 306 & $\begin{array}{l}\text { Wolinella succinogenes DSM 1740; Molybdenum cofactor biosynthesis protein A } \\
321,33 / 98(33 \%)(0.002)\end{array}$ \\
\hline CPT_0145 & 80 & $\begin{array}{l}\text { Helicobacter pylori.; Putative transposase OrfA } \\
210,31 / 69(44 \%)\left(3 e^{-09}\right)\end{array}$ \\
\hline CPT_0158 & 182 & $\begin{array}{l}\text { Campylobacter upsaliensis RM3195:; Thymidine kinase } \\
167,54 / 170(31 \%)\left(3 e^{-15}\right)\end{array}$ \\
\hline CPT_0164 & 181 & $\begin{array}{l}\text { Campylobacter fetus subsp. fetus 82-40.; Polysaccharide deacetylase } \\
305,40 / 151(26 \%)(0.006)\end{array}$ \\
\hline CPT_0165 & 212 & $\begin{array}{l}\text { Campylobacter fetus subsp. fetus 82-40; FAD-dependent thymidylate synthase } \\
206,113 / 200(56 \%)\left(4 e^{-52}\right)\end{array}$ \\
\hline CPT_0172 & 955 & $\begin{array}{l}\text { Campylobacter jejuni RM1221; Hypothetical protein CJE0595 } \\
426,71 / 176(40 \%)\left(3 \mathrm{e}^{-32}\right)\end{array}$ \\
\hline CPT_0191 & 171 & $\begin{array}{l}\text { Helicobacter hepaticus ATCC 51449; Hypothetical protein HH0767 } \\
188,61 / 187(32 \%)\left(6 \mathrm{e}^{-15}\right)\end{array}$ \\
\hline
\end{tabular}

\footnotetext{
${ }^{a}$ Length refers to the number of amino-acid residues in the CDS and are calculated from the genomic sequence

${ }^{\mathrm{b}}$ Gene product and putative function

${ }^{c}$ Identity and E values are from NCBI BLAST results, performed on $5^{\text {th }}$ January 2009
}

also a group III phage. Therefore, some genes would seem to be more widely spread in the sampled Campylobacter phage meta-genome than others.

The late transcription factor identified in both CP220 and CPt10 appears to be present in all of the group II phages tested but only in one of the group III phages. This may be indicative that these smaller phages are indeed different to the group II class, and have different regulatory factors controlling gene expression. The resolution of these questions requires that we sequence more genomes from these phages.

\section{Conclusions}

We have compared two novel genome sequences from independently isolated virulent Campylobacter phages and found them to be extremely similar to each other. Examination of further Campylobacter phages has revealed positive matches between a number of them and the two genomes sequenced in this paper, with the implication that the two sequenced phages are well adapted to their particular niche and that there are substantial selective pressures on these phages to maintain this particular genome configuration. It would suggest that phage genomes are as susceptible to selection for stability as they are for variability. Genome stability could be aided by the ability of these phages to display genotypic and phenotypic microvariation by harboring variable length DNA repeat sequences and expressing more than one form of structural protein. Concerted sequencing efforts may reveal the fate of these individual phage lineages and elucidate the extent of genetic flux within Campylobacter bacteriophages and between organisms sharing similar niches. However, for most organisms and certainly for Campylobacter, the number of sequenced phages is insufficient to ascertain how genome stability would play a role in competition with other phage that may have adopted alternative evolutionary strategies. 


\section{Methods \\ Campylobacter and Bacteriophage Storage and Growth Conditions}

Campylobacterstrains were stored at $-80^{\circ} \mathrm{C}$ in nutrient broth No. 2 (Oxoid, Basingstoke, UK) supplemented with $20 \%$ glycerol or Microbank vials (Prolab Diagnostics, Neston, United Kingdom). Strains were grown on blood agar base No. 2 (Oxoid) or Mueller-Hinton agar (Oxoid), supplemented with 5\% defibrinated horse blood (Oxoid), for 24-48 h under microaerobic conditions at $42^{\circ} \mathrm{C}$.

Bacteriophage were propagated using the whole plate lysis method, enumerated on C. jejuni strains HPC5 (CP220) or NCTC12668 (CPt10) and subsequently stored at $4^{\circ} \mathrm{C}$ in SM buffer as described previously [10].

\section{Bacteriophage Purification}

Bacteriophages were purified using caesium chloride equilibrium gradients [38]. The suspension was centrifuged at $264,000 \mathrm{~g}$ in a Beckman TLA 100.3 rotor at $4^{\circ} \mathrm{C}$ for 24 hours. Residual caesium chloride was removed using a Microcon 30,000 Da cut off column (Millipore, Watford, UK).

\section{Genome sequencing and analysis}

For CP220 a whole genome shotgun library was generated from the purified phage DNA. A shotgun sequencing approach was employed using subclone libraries of size fractionated (inserts $1.4-2 \mathrm{~kb}$ and 2-4 kb) phage DNA cloned into vectors pSMART and pUC19. The sequence was assembled from 2138 good quality reads (93\% pass rate) that were assembled using Phrap http://www.phrap.org into 3 contigs. A further 290 finishing reads were required to close the gaps in contigs and span low coverage regions by re-sequencing existing library clones or by oligo walks. The finished sequence, a 177493 bp contig consisting of 2376 reads, has a 10fold read coverage. The CPt10 genome was sequenced by 454 FLX pyrosequencing and assembled from 34265 sequence reads with an average read length of $173 \mathrm{bps}$ and constituting a theoretical 34-fold coverage, using the $454 /$ Roche Newbler assembly program. The gaps between these contigs were closed by directed PCR and the products sequenced with BigDye terminator chemistry on ABI3730 capillary sequencers.

Genome annotation was performed as previously described [39] using Artemis [40]. Insertion sequences were classified using the ISfinder database [41]. The genomes of $\mathrm{CP} 220$ and $\mathrm{CPt} 10$ were compared pair-wise using the Artemis Comparison Tool (ACT) [42].

\section{SDS-polyacrylamide gel electrophoresis (SDS-PAGE) and proteomics}

Purified phage at $\log _{10} 10 \mathrm{PFU} \mathrm{m} \mathrm{m}^{-1}$ were prepared for loading to a $4-15 \%$ gradient $\mathrm{Tris}-\mathrm{HCl}$ polyacrylamide
Ready Gel (Bio-Rad, Hemel Hempstead, UK) or 4-12\% Bis-Tris NuPAGE Novex gel (Invitrogen, Paisley, UK), using the SDS sample and gel running buffers supplied, according to the manufacturers' instructions. Gels were run at constant voltage of $200 \mathrm{v}$ for $35-50 \mathrm{~min}$ followed by staining, with colloidal coomassie blue, according to the manufacturers' instructions. Protein bands were excised from the gel, using a sterile scalpel, for mass spectroscopy analysis.

The gel slices were digested with trypsin, before undergoing electrospray ionization, with subsequent tandem MS/MS. The peptide fragment ions generated were analyzed using Mascot Daemon software [43] or MaxEnt3 maximum entropy software (Waters, Milford, USA).

\section{Repeat region PCR}

Primers used in the amplification of CP220 repeat sequences are shown in Additional file 4. The CP220 genomic sequence was used to design primers in unique flanking regions for each repeat region.

\section{PCR screening of Campylobacter typing phage}

Primers were designed using the CP220 and CPt10 genome sequences to amplify genes of interest including; insertion sequences, methylases and structural genes. Primer sequences are shown in Additional file 5 along with an indication of which genome sequence was used for the design of each primer pair using Primer3 [44].

Additional file 1: Supplementary Data - Table S1 - List of CDSs identified in phage CP220 and CPt10. Table S2 - Repeat region alignments in CP220 and CPt10.

Additional file 2: CP220, CPt10, Campylobacter jejuni and Campylobacter coli comparative codon usage.

Additional file 3: Distribution of CP220 and CPt10 CDSs in other phages of the UK Campylobacter typing scheme.

Additional file 4: Primers used in the analysis of CP220 repeat regions.

Additional file 5: Primers used in the comparative analysis of Campylobacter typing phages.

\section{Acknowledgements}

We thank Patricia Siguier (ISFinder) for help with defining the ends and classification of the IS elements. We thank the core sequencing and informatics teams at the Sanger Institute for their assistance and The Wellcome Trust for its support of the Sanger Institute Pathogen Genomics group.

\section{Author details}

'Division of Food Sciences, School of Biosciences, University of Nottingham, Sutton Bonington Campus, Loughborough, Leicestershire, LE12 5RD, UK. 2Department of Veterinary Medicine, University of Cambridge, Cambridge, CB3 OES, UK. ${ }^{3}$ Wellcome Trust Sanger Institute, Wellcome Trust Genome Campus, Hinxton, Cambridge, CB10 1SA, UK. ${ }^{4}$ Defence Science and Technology Laboratory, Porton Down, Salisbury, SP4 0JQ, UK. 


\section{Authors' contributions}

ART drafted the manuscript. JCY and NKP contributed to drafting the manuscript. ART, JCY, AES and NKP participated in the analysis of the genome sequences. PLC performed the SDS-PAGE analysis. NC performed the repeat sequence experiments. LC, KS and MQ performed the genome sequencing. NRT, DJM and IFC conceived of the study and participated in its design and coordination and critically reviewed the manuscript. All authors read and approved the final manuscript.

Received: 7 September 2009 Accepted: 30 March 2010 Published: 30 March 2010

\section{References}

1. Hendrix RW, Smith MC, Burns RN, Ford ME, Hatfull GF: Evolutionary relationships among diverse bacteriophages and prophages: All the world's a phage. PNAS 1999, 96:2192-2197.

2. Denou E, Bruttin A, Barretto C, Ngom-Bru C, Brussow H, Zuber S: T4 phages against Escherichia coli diarrhea: potential and problems. Virology 2009, 388:21-30.

3. Hatfull GF, Jacobs-Sera D, Lawrence JG, Pope WH, Russell DA, Ko CC, Weber RJ, Patel MC, Germane KL, Edgar RH, et al: Comparative genomic analysis of sixty mycobacteriophage genomes: Genome clustering, gene acquisition and gene size. J Mol Biol 2010.

4. Adak GK, Meakins SM, Yip H, Lopman BA, O'Brien SJ: Disease risks from foods, England and Wales, 1996-2000. Emerg Infect Dis 2005, 11:365-372.

5. Allos BM: Campylobacter jejuni Infections: update on emerging issues and trends. Clin Infect Dis 2001, 32:1201-1206.

6. Miller WG, Mandrell RE: Prevalence of Campylobacter in the food and water supply: incidence, outbreaks, isolation and detection. Campylobacter: Molecular and Cellular Biology Norfolk: Horizon Scientific PressKetley JM, Konkel ME 2005, 101-163.

7. Overell JR, Willison HJ: Recent developments in Miller Fisher syndrome and related disorders. Curr Opin Neurol 2005, 18:562-566.

8. Yuki N, Koga M: Bacterial infections in Guillain-Barre and Fisher syndromes. Curr Opin Neurol 2006, 19:451-457.

9. Ternhag A, Törner A, Svensson Å, Ekdahl K, Giesecke J: Short- and longterm effects of bacterial gastrointestinal infections. Emerg Infect Dis 2008, 14:143-148.

10. Loc Carrillo C, Atterbury RJ, El-Shibiny A, Connerton PL, Dillon E, Scott A, Connerton IF: Bacteriophage therapy to reduce Campylobacter jejuni colonization of broiler chickens. Appl Environ Microbiol 2005, 71:6554-6563.

11. Wagenaar JA, Bergen MA, Mueller MA, Wassenaar TM, Carlton RM: Phage therapy reduces Campylobacter jejuni colonization in broilers. Vet Microbiol 2005, 109:275-283.

12. Atterbury RJ, Connerton PL, Dodd CER, Rees CED, Connerton IF: Application of host-specific bacteriophages to the surface of chicken skin leads to a reduction in recovery of Campylobacter jejuni. Appl Environ Microbiol 2003, 69:6302-6306.

13. Frost JA, Kramer JM, Gillanders SA: Phage typing of Campylobacter jejuni and Campylobacter coli and its use as an adjunct to serotyping. Epidemiol Infect 1999, 123:47-55.

14. Grajewski BA, Kusek JW, Gelfand HM: Development of a bacteriophage typing system for Campylobacter jejuni and Campylobacter coli. I Clin Microbiol 1985, 22:13-18.

15. Ackermann HW: 5500 Phages examined in the electron microscope. Arch Virol 2007, 152:227-243.

16. Atterbury RJ, Connerton PL, Dodd CE, Rees CE, Connerton IF: Isolation and characterization of Campylobacter bacteriophages from retail poultry. Appl Environ Microbiol 2003, 69:4511-4518.

17. Sails AD, Wareing DR, Bolton FJ, Fox AJ, Curry A: Characterisation of 16 Campylobacter jejuni and C. coli typing bacteriophages. J Med Microbiol 1998, 47:123-128.

18. Loc Carrillo CM, Connerton PL, Pearson T, Connerton IF: Free-range layer chickens as a source of Campylobacter bacteriophage. Antonie Van Leeuwenhoek 2007, 92:275-284.

19. Connerton IF, Connerton PL, Barrow P, Seal BS, Atterbury RJ: Bacteriophage therapy and Campylobacter. Campylobacter Washington D.C.: ASM PressNachamkin I, Szymanski CM, Blaser MJ , 3 2008, 679-693.

20. El-Shibiny A, Scott A, Timms A, Metawea Y, Connerton P, Connerton I: Application of a group II Campylobacter bacteriophage to reduce strains of Campylobacter jejuni and Campylobacter coli colonizing broiler chickens. J Food Prot 2009, 72:733-740.

21. Salama S, Bolton FJ, Hutchinson DN: Improved method for the isolation of Campylobacter jejuni and Campylobacter coli bacteriophages. Lett Appl Microbiol 1989, 8:5-7.

22. CampyDB. [http://xbase.bham.ac.uk/campydb/].

23. Villegas A, She YM, Kropinski AM, Lingohr EJ, Mazzocco A, Ojha S, Waddell TE, Ackermann HW, Moyles DM, Ahmed R, et al: The genome and proteome of a virulent Escherichia coli 0157:H7 bacteriophage closely resembling Salmonella phage Felix O1. Virol I 2009, 6:41.

24. Kwan T, Liu J, DuBow M, Gros P, Pelletier J: The complete genomes and proteomes of 27 Staphylococcus aureus bacteriophages. PNAS 2005, 102:5174-5179.

25. Lobry JR: Asymmetric substitution patterns in the two DNA strands of bacteria. Mol Biol Evol 1996, 13:660-665.

26. Miller ES, Kutter E, Mosig G, Arisaka F, Kunisawa T, Ruger W: Bacteriophage T4 genome. Microbiol Mol Biol Rev 2003, 67:86-156, table.

27. Liu C, Heath $L S$, Turnbough $C L \mathrm{Jr}$ : Regulation of pyrBl operon expression in Escherichia coli by UTP-sensitive reiterative RNA synthesis during transcriptional initiation. Genes Dev 1994, 8:2904-2912.

28. Penno C, Sansonetti P, Parsot C: Frameshifting by transcriptional slippage is involved in production of MxiE, the transcription activator regulated by the activity of the type III secretion apparatus in Shigella flexneri. Mol Microbiol 2005, 56:204-214.

29. Wang SC, Frey PA: S-adenosylmethionine as an oxidant: the radical SAM superfamily. Trends Biochem Sci 2007, 32:101-110.

30. EMBL InterPro Database: IPR007197 Radical SAM. [http://www.ebi.ac.uk/ interpro/IEntry?ac=IPR007197].

31. Alam Tl, Draper B, Kondabagil K, Rentas FJ, Ghosh-Kumar M, Sun S, Rossmann MG, Rao VB: The headful packaging nuclease of bacteriophage T4. Mol Microbiol 2008, 69:1180-1190.

32. Russell RL, Huskey RJ: Partial exclusion between T-even bacteriophages: an incipient genetic isolation mechanism. Genetics 1974, 78:989-1014.

33. Brok-Volchanskaya VS, Kadyrov FA, Sivogrivov DE, Kolosov PM, Sokolov AS, Shlyapnikov MG, Kryukov VM, Granovsky IE: Phage T4 SegB protein is a homing endonuclease required for the preferred inheritance of T4 tRNA gene region occurring in co-infection with a related phage. Nucleic Acids Res 2008, 36:2094-2105.

34. Rohwer F: Global phage diversity. Cell 2003, 113:141

35. Comeau AM, Bertrand C, Letarov A, Tetart F, Krisch HM: Modular architecture of the T4 phage superfamily: a conserved core genome and a plastic periphery. Virology 2007, 362:384-396.

36. Lunt MR, Siebke JC, Burton K: Glucosylated nucleotide sequences from T2-bacteriophage deoxyribonucleic acid. Biochem J 1964, 92:27-36.

37. Luria SE, Human ML: A nonhereditary, host-induced variation of bacterial viruses. J Bacteriol 1952, 64:557-569.

38. Sambrook J, Russell DW: Molecular cloning: a laboratory manual Cold Spring Harbor, N.Y: Cold Spring Harbor Laboratory Press 2001.

39. Parkhill J, Wren BW, Mungall K, Ketley JM, Churcher C, Basham D, Chillingworth T, Davies RM, Feltwell T, Holroyd S, et al: The genome sequence of the food-borne pathogen Campylobacter jejuni reveals hypervariable sequences. Nature 2000, 403:665-668.

40. Rutherford K, Parkhill J, Crook J, Horsnell T, Rice P, Rajandream MA, Barrell B: Artemis: sequence visualization and annotation. Bioinformatics 2000, 16:944-945.

41. ISfinder Database. [http://www-is.biotoul.fr/is.html].

42. Carver TJ, Rutherford KM, Berriman M, Rajandream MA, Barrell BG, Parkhill J: ACT: the Artemis Comparison Tool. Bioinformatics 2005, 21:3422-3423.

43. Perkins DN, Pappin DJ, Creasy DM, Cottrell JS: Probability-based protein identification by searching sequence databases using mass spectrometry data. Electrophoresis 1999, 20:3551-3567.

44. Rozen S, Skaletsky H: Primer3 on the WWW for general users and for biologist programmers. Methods Mol Biol 2000, 132:365-386.

45. The Codon Usage Database. [http://www.kazusa.or.jp/codon/].

doi:10.1186/1471-2164-11-214

Cite this article as: Timms et al:: Evidence for a lineage of virulent bacteriophages that target Campylobacter. BMC Genomics 2010 11:214. 